\title{
Über den Mechanismus der polarographischen Reduktion aromatischer Nitroverbindungen
}

\author{
Von \\ L. Holleck und B. Kastening \\ Chemisches Institut der Hochschule Bamberg (Deutschland) \\ Herrn Professor Dr. I. Tachi gewidmet.
}

(Eingegangen am 21. Dez. 1962)

Für die Lage der Reduktionspotentiale bei aromatischen Nitroverbindungen sind fast ausnahmslos kinetische Einflüsse maßgeblich ; die Reduktion verläuft unter sehr erheblicher Überspannung. Rein thermodynamisch bedingte Vorgänge treten nur als Teilvorgänge unter bestimmten Umständen in Erscheinung. Es wird ein Überblick gegeben über die verschiedenen Mechanismen, die in den einzelnen $\mathrm{pH}$-Bereichen maßgeblich sind. Im alkalischen Bereich und in protonenarmen Medien folgt dem reversiblen Primärvorgang (Aufnahme des ersten Elektrons) als geschwindigkeitsbestimmender Schritt der Durchtritt des zweiten Elektrons ; verschiedene Einflüsse auf die Durchtrittsgeschwindigkeit werden besprochen (Wirkung von Substituenten, der Lösungszusammensetzung, des Grenzflächenzustands, insbesondere der Adsorption von Fremdstoffen = Inhibitoren). In Gegenwart grenzflächenaktiver Stoffe treten in wässrig-neutralen Lösungen zu den genannten Schritten Lösungsreaktionen hinzu, die den langsamen Durchtritt des zweiten Elektrons umgehen. In Abwesenheit solcher Stoffe sind im Bereich pH 0 bis 10 offenbar sowohl Durchtrittsreaktionen wie auch im adsorbierten Zustand verlaufende Protonenübergänge am geschwindigkeitsbestimmenden Vorgang beteiligt; hier lässt sich die Geschwindigkeit formal durch eine $\mathrm{pH}$ - und potentialabhängige Konstante beschreiben. Diese heterogenen Protonenübergänge werden durch Inhibitoren ausgeschaltet, sodass dann in sauren Lösungen der Elektronenübergang auf das durch Protonenaufnahme im Lösungsgleichgewicht entstehende Kation bestimmend wird.

Die ersten Untersuchungen über die polarographische Reduktion aromatischer Nitroverbindungen erfolgten in der Frühzeit der Polarographie und sind ein Verdienst der japanischen Schule, hauptsächlich von M. Shikat a und I. Tachi i). Danach wurden diese Vorgänge in zahlreichen Arbeiten verschiedener Autoren untersucht, auf die in diesem Rahmen nicht eingegangen werden kann. In dieser Arbeit sollen die wesentlichen Ergebnisse einer Reihe vorangehender eigener Publikationen ${ }^{2-17}$, z.T. gemeinsam mit H. J. Exner und anderen, soweit sie den Reduktionsmechanismus betreffen, zusammengefasst und durch bislang unveröffentliche Resultate ergänzt werden. Auf einige neuere Arbeiten anderer Autoren wird im Zusammenhang mit den einzelnen Erscheinungen hingewiesen.

In den vorangehenden Arbeiten $^{2-17)}$ wurde die polarographische NitrogruppenReduktion unter verschiedenen Aspekten beleuchtet. Bei diesen Untersuchungen gilt das Interesse nicht nur den Reaktionsweisen gerade dieser Stoffklasse, sondern vor allem den verschiedenen Reaktionstypen selbst, die sich im Verhalten anderer funktioneller Gruppen wiederfinden. Es seien genannt: Reversibilität von Redoxsystemen, irreversible Vorgänge, 
Durchtrittskinetik, vor- und nachgelagerte Lösungsreaktionen, katalytische Prozesse, Eigenadsorption des Depolarisators, Auswirkung adsorbierter Fremdstoffe, chemische Grenzflächenreaktionen, ferner die Einflüsse der Konstitution (Wirkung von Substituenten), des Lösungsmittels und der Lösungszusammensetzung u. a. m.

Die im folgenden angeführten Einzelheiten des Mechanismus können für alle aromatischen Nitroverbindungen, d.h. Abkömmlinge des Nitrobenzols, als gültig angesehen werden; dabei ist jedoch darauf hinzuweisen, dass u. U. Komplikationen eintreten, z.B. bei dissoziierbaren Verbindungen (Nitrophenol, Nitrobenzoesäure ${ }^{3)}$, Nitranilin ${ }^{7)}$ ) oder solchen, die weitere reduzierbare Gruppen tragen $\left(\mathrm{Di}^{-5}{ }^{5}\right.$ und Trinitroverbindungen $\left.{ }^{6}\right)$. Sie sind nicht ohne weiteres auf aliphatische Nitroverbindungen $\mathrm{zu}$ übertragen ${ }^{18,19,20)}$, finden sich jedoch bei aromaten-ähnlichen Stoffen wieder $\left(\omega\right.$-Nitrostyrol ${ }^{15)}$, Nitronaphthalin ${ }^{17)}$, Nitropyridin ${ }^{21)}$ ).

Ausgehend von der Frage nach der thermodynamischen Reversibilität der Vorgänge und dem Ausmaß der Irreversibilität der Gesamtreduktion soll im weiteren ein Überblick über die Mechanismen in den einzelnen $\mathrm{pH}-$ Bereichen gegeben werden.

\section{Thermodynamische Betrachtungen}

Die Endprodukte der Reduktion von Nitroverbindungen sind gewöhnlich die entsprechenden Hydroxylamino- oder Aminoverbindungen. (Bei präparativer Arbeitsweise können ausserdem Stoffe der Azo-Klasse entstehen; näheren Einblick in diese Verhältnisse haben Kemula u. Kubli k ${ }^{22}$ durch Untersuchungen am hängenden Tropfen gewonnen.) Als Gesamtvorgang sind diese Prozesse stets irreversibel, verlaufen also nicht bei den ihnen thermodynamisch zukommenden Redoxpotentialen. Hierzu gehört auch die Reduktion bis zur Nitroso-Zwischenstufe. Wenngleich sich die thermodynamischen Redoxpotentiale nicht genau angeben lassen, da die Werte der hierzu benötigten thermodynamischen Funktionen usw. in der Literatur nur unvollkommen sind und man daher auf Schätzungen angewiesen ist, kann die Grössenordnung der auf diese Weise abgeschätzten Werte doch soweit als sicher gelten, dass das Ausmass der Irreversibilität, d.h. der Überspannung, hieraus deutlich wird. In Tab. 1 sind solche Schätzwerte für die Freie Reaktionsenthalpie und die sich hieraus errechnenden Redox-Normalpotentiale angegeben und den polarographischen Halbstufenpotentialen (mittlerer Wert, der sowohl für die 4-Elektronen-Reduktion zum Hydroxylamin-Derivat wie auch für die 6-ElektronenReduktion zum Amin gilt) gegenübergestellt. (Vor- bzw. nachgelagerte Säure-Base-

Tabelle 1. Normalwerte der Freien Reaktionsenthalpie $\Delta^{N} G$ und des Redoxpotentials ${ }^{N} \varepsilon$ für die Reduktion aromatischer Nitroverbindungen zur Nitroso-, Hydroxylaminound Amin-Stufe (aus z.T. abgeschätzten Werten berechnet) sowie polarographisches Halbstufenpotential $\varepsilon_{1 / 2}$ (Mittelwert einiger Nitroverbindungen).

\begin{tabular}{c|c|c|c}
\hline & $\begin{array}{c}\Delta^{N} G \\
(\mathrm{kcal} / \mathrm{mol})\end{array}$ & $\begin{array}{c}N^{N} \varepsilon \\
(\text { Volt, GKE*) }\end{array}$ & $\begin{array}{c}\varepsilon_{1 / 2} \\
(\text { Volt, GKE*) }\end{array}$ \\
\hline $\mathrm{R}-\mathrm{NO}_{2}+2 \mathrm{e}+2 \mathrm{H}^{+}$ \\
$\stackrel{\mathrm{R}-\mathrm{NO}+\mathrm{H}_{2} \mathrm{O}}{\longrightarrow}$ & $-46+46 \cdot \varepsilon+2,7 \mathrm{pH}$ & $+1,0-0,059 \mathrm{pH}$ & - \\
$\mathrm{R}-\mathrm{NO}_{2}+4 \mathrm{e}+4 \mathrm{H}^{+}$ \\
$\stackrel{\mathrm{R}-\mathrm{NHOH}+\mathrm{H}_{2} \mathrm{O}}{\longrightarrow}$ & $-60+92 \cdot \varepsilon+5,4 \mathrm{pH}$ & $+0,65-0,059 \mathrm{pH}$ & \\
$\mathrm{R}-\mathrm{NO}_{2}+6 \mathrm{e}+6 \mathrm{H}^{+}$ & $-81+138 \cdot \varepsilon+8,1 \mathrm{pH}$ & $+0,6-0,059 \mathrm{pH}$ & $-0,1-0,07 \mathrm{pH}$ \\
\hline
\end{tabular}

*) $\mathrm{GKE}=$ gesättigte Kalomelektrode 
Gleichgewichte bei der Bildung von Formen der Reduktionsprodukte wie R-NHO${ }^{-}$, $\mathrm{R}-\mathrm{NH}_{2} \mathrm{OH}^{+}, \mathrm{R}-\mathrm{NH}_{3}{ }^{+}$oder an anderen funktionellen Gruppen des Depolarisators, wie bei Nitrophenol, Nitranilin, Nitrobenzoesäure usw., blieben hierbei unberücksichtigt.) Hieraus ergeben sich, je nach pH-W€rt, für die Überspannung Werte von etwa 0,7 bis 0,9 Volt.

Reversibilität der Redoxsysteme tritt tatsächlich nur bei Teilprozessen der Gesamtreduktion in Erscheinung, nämlich entweder bei der Untersuchung von Zwischenprodukten (hierzu gehören die reversiblen Systeme Nitrosobenzol/Phenylhydroxylamin und Chinonimin/Aminophenol) oder bei Untersuchung der Nitroverbindung unter ganz bestimmten Umständen; letzteres betrifft den unter Bildung eines relativ stabilen Radikalanions verlaufenden einelektronigen Primärschritt, der in wässrig-alkalischer Lösung in Gegenwart grenzflächenaktiver Stoffe (Inhibitoren) ${ }^{2,3,9)}$ sowie in gewissen nichtwässrigen Lösungsmitteln ${ }^{12,13,23,24)} \mathrm{zu}$ beobachten ist :

$$
\mathrm{R}-\mathrm{NO}_{2}+\mathrm{e} \rightleftarrows \mathrm{R}-\mathrm{NO}_{2}^{-}
$$

Die Untersuchung der zugehörigen einelektronigen Stufe und die Zuordnung zum reversiblen Primärvorgang (I) erfolgte durch Holleck u. Exne r ${ }^{2,3} *$.

Die Reversibilität dieses Vorgangs haben wir auf verschiedene Weise nachweisen können; das zugehörige RedoxNormalpotential und der Normalwert der Freien Reaktionsenthalpie ist für einige Verbindungen in Tab. 2 verzeichnet.

Die - abgesehen vom Primärvorgang -sonst stets festgestellte Irreversibilität der Reduktionsprozesse, ergänzt durch Untersuchungen der Oxydation der Reduktionsprodukte an Kohlepastenelektroden $^{27)}$, gibt daher zur Untersuchung des Mechanismus und der für

Tabelle 2. Normalwerte des Redoxpotentials und der Freien Reaktionsenthalpie für den einelektronigen Primärvorgang (I) bei verschieden substituierten Nitrobenzolen (in wässriger Lösung experimentell ermittelt).

\begin{tabular}{l|c|c}
\hline Substituent & $\begin{array}{c}N_{\varepsilon} \\
(\text { Volt, GKE) }\end{array}$ & $\begin{array}{c}\Delta^{N} G \\
(\mathrm{kcal} / \mathrm{mol})\end{array}$ \\
\hline $\mathrm{m}-\mathrm{NH}_{2}$ & $-0,705$ & $+16,2+23 \cdot \varepsilon$ \\
$\mathrm{m}-\mathrm{CH}_{3}$ & $-0,705$ & $+16,2+23 \cdot \varepsilon$ \\
$\mathrm{m}-\mathrm{Cl}$ & $-0,635$ & $+14,6+23 \cdot \varepsilon$ \\
\hline $\mathrm{p}-\mathrm{NH}_{2}$ & $-0,825$ & $+19,0+23 \cdot \varepsilon$ \\
$\mathrm{p}-\mathrm{CH}_{3}$ & $-0,73$ & $+16,8+23 \cdot \varepsilon$ \\
$\mathrm{p}-\mathrm{Cl}$ & $-0,675$ & $+15,5+23 \cdot \varepsilon$ \\
\hline
\end{tabular}
die kinetische Hemmung massgeblichen Teilschritte Anlass. Hierüber wird im folgenden Abschnitt berichtet.

\section{Mechanismus und Kinetik}

Bei einem Vorgang, bei dem insgesamt vier oder gar sechs Elektronen zu übertragen sind und bei dem überdies eine oder zwei N-O-Bindungen gelöst werden, ist es von vornherein einleuchtend, dass der Gesamtprozess nur in mehreren Einzelschritten ablaufen kann; dies wird auch durch Untersuchungen mittels oszillographischer Polarographie bestätigt ${ }^{28}$. Die Geschwindigkeit des Gesamtvorgangs und damit das Reduktionspotential werden dabei einerseits durch die Geschwindigkeit des langsamsten Teilvorgangs und andererseits durch die diesem etwa vorgelagerten Gleichgewichte, etwa den oben bezeichneten reversiblen Primärvorgang (I) oder Protonenübergänge, bestimmt. Welches

*) Eine ähnliche polarographische Stromspannungskurve hatten-ohne den Effekt näher zu deutenschon Heyrovský, Šorm u. Forej $\mathrm{t}^{25}$ ) in pyridinhaltigen Lösungen von Nitrobenzol erhalten.-Zur Wirkung grenzflächenaktiver Stoffe auf die Nitrogruppen-Reduktion vergl. auch Strassner u. Delahay ${ }^{26)}$. 
der geschwindigkeitsbestimmende Teilschritt ist, hängt hauptsächlich vom Protonenangebot der Lösung ab; die Besprechung der einzelnen Mechanismen wird daher im folgenden nach dieser Eigenschaft der Lösung unterteilt.

\section{Protonenarme Medien}

In solchen Medien, in denen das Protonenangebot gering ist, wird die Reduktion durch den einelektronigen Primärschritt (I) eingeleitet. Dies gilt sowohl in wässrigalkalischen Lösungen $(\mathrm{pH}>10 \text {, bei Gegenwart von Inhibitoren } \mathrm{pH}>6)^{2,3)}$ wie in gewissen nichtwässrigen Lösungsmitteln ${ }^{12,13,23,24)}$. Als geschwindigkeitsbestimmender Folgeschritt folgt dann die Aufnahme des zweiten Elektrons durch das primär gebildete Radikal$\operatorname{anion}^{11)}$ :

$$
\mathrm{R}-\stackrel{\times}{\mathrm{NO}_{2}}{ }^{-}+\mathrm{e} \longrightarrow \mathrm{R}_{-} \mathrm{NO}_{2}^{--}
$$

Die weiteren Folgeschritte verlaufen rasch bis zum jeweiligen Endprodukt (R-NHOH oder $\mathrm{R}-\mathrm{NH}_{2}$ ) ; welche Stufen dabei durchlaufen werden, lässt sich nicht ohne weiteres feststellen. Das gebildete Ion ist die hydratisierte und dissoziierte Nitrosoverbindung; es ist daher denkbar, dass es durch Reaktion mit Lösungsmittel in die Nitrosoverbindung überführt wird, die dann rasch weiterreduziert wird.

Die Geschwindigkeit des Vorgangs (II) und verschiedene Faktoren, die hierauf Einfluss nehmen, haben wir näher untersucht. Dabei ist zu beachten, dass auch der Vorgang (II), ebenso wie der Primärprozess (I), noch kein eigentlicher Elementarschritt ist, sondern sich aus dem Adsorptionsprozess des Radikalanions (einem Gleichgewicht) und dem Úbergang des Elektrons im adsorbierten Zustand (dem langsamen Schritt) zusammensetzt ${ }^{11,16)}$. Die Gesamtreduktion ist also wie folgt zu formulieren :

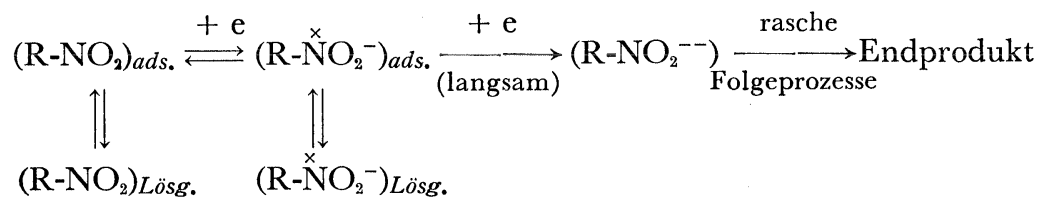

Für ein solches Reaktionsschema lässt sich der Verlauf der polarographischen Stromspannungskurven durch den Ausdruck ${ }^{11)}$

$$
\frac{i}{i^{*}}=\frac{1}{1+\lambda}+\frac{3+4 \lambda}{1+\lambda} \cdot \Psi(\chi)
$$

beschreiben (für insgesamt 4-elektronige Reduktion; bei Amin-Bildung sind statt " 3 " bzw. "4" die Zahlen "5" bzw. "6" zu setzen). Darin bedeuten

$i=$ Stromstärke beim Potential $\varepsilon$

$i^{*}=$ Diffusionsgrenzstrom eines Ein-Elektronen-Vorgangs

$\lambda=\exp \left[\frac{F\left(\varepsilon-{ }^{N} \varepsilon\right)}{R T}\right] ;{ }^{N} \varepsilon=$ Redox-Normalpotential des Primärvorgangs (I)

$\Psi(\chi)=$ Koutecký-Funktion ${ }^{29}$

$\chi=\frac{k \cdot r}{1+\lambda} \cdot \sqrt{\frac{12 t}{7 D}}$

$k=k\left({ }^{N} \varepsilon\right) \cdot \lambda^{-\alpha}=$ Geschwindigkeitskonstante für den Durchtritt des zweiten Elektrons (II)

$\alpha=$ Durchtrittsfaktor

$\gamma=$ Adsorptionskoeffizient des Primärprodukts = Radikalanions (Verhältnis von Grenzflächen- zu Lösungskonzentration in Elektrodennähe) 
$t=$ Tropfzeit $; D=$ Diffusionskoeffizient

Bezeichnet man mit $k_{0}=r \cdot k\left({ }^{N} \varepsilon\right)$ die formale Geschwindigkeitskonstante beim RedoxNormalpotential des Primärvorgangs, so lassen sich die resultierenden Stromspannungskurven anschaulicher so beschreiben:

Ist $k_{0} \ll 10^{-3} \mathrm{~cm} / \mathrm{sec}$, so erscheint beim Potential ${ }^{N} \varepsilon$ nur die reversible 1-ElektronenStufe; bei negativeren Potentialen erscheint dann eine zweite, irreversible Stufe. Ist $k_{0} \approx 10^{-3} \mathrm{~cm} / \mathrm{sec}$, so erfolgt die Gesamtreduktion in einer einheitlichen Stufe, deren Halbstufenpotential etwa mit ${ }^{N} \varepsilon$ übereinstimmt. Ist schließlich $k_{0}>10^{-3} \mathrm{~cm} / \mathrm{sec}$, so erscheint ebenfalls eine ungeteilte Gesamtstufe, deren Halbstufenpotential positiver als ${ }^{N} \varepsilon$ ist.

Der Wert von $k_{0}$ und demnach Form und Lage der Stufen hängt nun von verschiedenen Faktoren ab: von der Art der Nitroverbindung selbst, vom Lösungsmittel, von der Lösungszusammensetzung und vom Zustand der Grenzfläche. Diese Einflüsse sollen im folgenden diskutiert werden.

Die Substitution einer zweiten funktionellen Gruppe am Benzolkern außer der Nitrogruppe beeinflusst zunächst-durch Änderung der Elektronenniveaus und damit der Resonanzenergien sowie der Solvatationsverhältnisse-das chemische Potential von Nitroverbindung und Radikalanion in Lösung. Dies hat eine Verschiebung des RedoxNormalpotentials zu Folge, wie aus Tab. 2 hervorgeht. Diese Änderung der chemischen Potentiale in Lösung sowie die durch den Substituenten bedingte Änderung der Adsorptionskräfte beider Teilchen bewirken weiterhin Verschiebungen der beiden Adsorptionsgleichgewichte. Zu diesen beiden Einflüssen des Substituenten auf die Geschwindigkeit tritt diejenige auf den Durchtrittsvorgang selbst, die eine Folge der veränderten Elektronendichte-Verteilung sowie der sterischen Anordnung in der Adsorptionsschicht ist. Die verschiedenen Einflüsse heben sich offenbar z.T. heraus, da die formale Konstante $k_{0}$, nach Tab. 3 (Spalte 3), nur geringe Unterschiede aufweist und die Abweichung des Halbstufenpotentials (Spalte 4) vom Redox-Normalpotential nur zwischen 15 und $30 \mathrm{mV}$ schwankt. (In Lösungen höheren Salzgehalts sind die Unterschiede größer: Bei der Ionenstärke $I=1 \mathrm{schwankt} k_{0}$ maximal um etwa eine Größenordnung; die Abweichung zwischen ${ }^{N} \varepsilon$ und $\varepsilon_{1 / 2}$ schwankt zwischen 35 und $60 \mathrm{mV}$.)

Tabelle 3. Werte der formalen Geschwindigkeitskonstanten $k_{0}$ und des Durchtrittsfaktors $\alpha$ für den Sekundärprozess (II) sowie Halbstufenpotentiale, ohne und mit Inhibitor, für einige substituierte Nitrobenzole in wässriger Lösung ( $10 \%$ Methanol, $0,02 n \mathrm{NaOH}$, $0,08 n \mathrm{KCl})$.

\begin{tabular}{|c|c|c|c|c|c|c|c|}
\hline & \multirow{2}{*}{$\begin{array}{c}N_{\varepsilon} \\
(\mathrm{V}, \mathrm{GKE})\end{array}$} & \multicolumn{2}{|c|}{ ohne Inhibitor } & \multicolumn{3}{|c|}{ mit $0,05 \%$ Campher } & \multirow[b]{2}{*}{$\Delta \log k_{0}$} \\
\hline & & $\left|\begin{array}{c}\log k_{0} \\
\left(k_{0} \text { in } \mathrm{cm} / \mathrm{sec}\right)\end{array}\right|$ & $\begin{array}{c}\varepsilon_{1 / 2} \\
(V, G K E)\end{array}$ & $\left|\begin{array}{c}\log k_{0} \\
\left(k_{0} \text { in } \mathrm{cm} / \mathrm{sec}\right)\end{array}\right|$ & $\begin{array}{c}\varepsilon_{1 / 2} \\
(2 . \text { Stufe }) \\
(V, G K E)\end{array}$ & $\alpha$ & \\
\hline $\mathrm{m}-\mathrm{NH}_{2}$ & $-0,705$ & $-2,41$ & $-0,69$ & $-8,20$ & $-1,19$ & 0,635 & 5,79 \\
\hline $\mathrm{m}-\mathrm{CH}_{3}$ & $-0,705$ & $-2,30$ & $-0,685$ & $-7,25$ & $-1,15$ & 0,58 & 4,95 \\
\hline $\mathrm{m}-\mathrm{Cl}$ & $-0,635$ & $-2,10$ & $-0,605$ & $-5,87$ & $-0,975$ & 0,51 & 3,77 \\
\hline $\mathrm{p}-\mathrm{NH}_{2}$ & $-0,825$ & $-2,31$ & $-0,81$ & $\sim-10^{*}$ & $\sim-1,28 *$ & $\sim 1,0^{*}$ & $\sim 8^{*}$ \\
\hline $\mathrm{p}-\mathrm{CH}_{3}$ & $-0,73$ & $-2,41$ & $-0,715$ & $-8,22$ & $-1,23$ & 0,62 & 5,81 \\
\hline $\mathrm{p}-\mathrm{Cl}$ & $-0,675$ & $-2,24$ & $-0,65$ & $-6,15$ & $-1,09$ & 0,455 & 3,91 \\
\hline
\end{tabular}

*) unsichere Werte, da bereits bei - 1,245 V Desorption des Inhibitors erfolgt, sodass nur der Stufenfuss auswertbar ist. 
Auch das Lösungsmittel beeinflusst infolge geänderter Solvatationsverhältnisse die Lage der Adsorptionsgleichgewichte sowie ${ }^{N} \varepsilon$ und bewirkt daher auch eine Änderung von $k_{0}$. Starke Dielektrika (Wasser) erniedrigen das chemische Potential der Ionenform in Lösung, während organische Lösungsmittel dasjenige der Nitroverbindung herabsetzen, wie sich schon in der Löslichkeit zu erkennen gibt. Infolgedessen wird ${ }^{N} \varepsilon$ um so negativer, je mehr sich der Charakter des Lösungsmittels von dem des Wassers entfernt, wie aus Tab. 4 hervorgeht. Neben dem Einfluss auf die vorgelagerten Gleichgewichte wirkt sich

Tabelle 4. Redox-Normalpotentiale für den Primärvorgang (I) und Halbstufenpotentiale von $p$-Nitrochlorbenzol in verschiedenen Lösungsmitteln. (Volt, GKE).

\begin{tabular}{l|c|c|c}
\hline & $N_{\varepsilon}$ & $\begin{array}{c}\varepsilon_{1 / 2} \\
\text { Gesamtstufe }\end{array}$ & $\begin{array}{c}\varepsilon_{1 / 2} \\
2 . \text { Stufe }\end{array}$ \\
\hline $\begin{array}{c}\text { Wasser } \\
(10 \% \text { Methanol, } 0,02 n \\
\text { NaOH } 0,08 n \mathrm{KCl})\end{array}$ & $-0,675$ & $-0,65$ & - \\
\hline $\begin{array}{c}\text { Methanol } \\
\left(0,5 n \mathrm{CH}_{3} \mathrm{ONa}\right)\end{array}$ & $-0,78$ & $-0,84$ & $-1,18$ \\
\hline $\begin{array}{c}\text { Acetonitril } \\
\left(0,1 n \mathrm{NaJ}^{13}\right)\end{array}$ & $-0,965$ & - & $-1,41$ \\
\hline $\begin{array}{c}\text { Dimethylformamid } \\
\left(0,1 n \mathrm{NaJ}^{13)}\right.\end{array}$ & $-0,945$ & - & $-1,52$ \\
\hline \begin{tabular}{c}
$\left(0,1 n \mathrm{CsJ}^{2}\right)$ \\
\hline$\left(0,1 n \mathrm{~N}\left[\mathrm{C}_{2} \mathrm{H}_{5}\right]_{4} \mathrm{~J}\right)$
\end{tabular} & $-0,985$ & - & $-1,87$ \\
\hline
\end{tabular}

das Lösungsmittel auf $k_{0}$ auch noch durch die Veränderung der Grenzschicht-Struktur aus. Beide Einflüsse wirken zusammen $: k_{0}$ wird immer kleiner und damit die Weiterreduktion immer stärker zu negativen Potentialen verschoben, wie Tab. 4 zeigt, je mehr man sich vom Wasser entfernt.

Andere Lösungsbestand teile, wie Leitsalze und sonstige Zusätze, üben ebenfalls einen Einfluss aus. Die Leitsalzkonzentration kann die vorgelagerten Gleichgewichte durch Änderung der chemischen Potentiale verschieben. In wässriger Lösung ist die Verschiebung des Redox-Normalpotentials nur gering (ca. 5 bis $10 \mathrm{mV}$ ). In schwachen Dielektrika ist diese Wirkung jedoch stärker. Untersuchungen in Dimethylformamid ${ }^{13)}$ mit verschiedenen Leitsalzen ergaben eine merkliche Abhängigkeit von der Art des Kations (vergl. Tab. 4), sodass diese Wirkung als Folge einer Ionenpaarbildung zu interpretieren ist: je kleiner das Kation, um so mehr verschiebt sich das Gleichgewicht zugunsten der Ionenform und das Redoxpotential wandert zu positiveren Werten. Erheblicher ist die Wirkung des Leitsalzes-auch in wässriger Lösung, wie Tab. 5 zeigt - auf die Geschwindigkeit der Sekundärreduktion $\left(k_{0}\right)$, die nicht nur auf der Verschiebung der vorgelagerten Gleichgewichte, auch der Adsorptionsgleichgewichte, beruht, sondern auch eine Folge der veränderten Grenzschichtstruktur ist. Auf die beschleunigende Wirkung erhöhter Salzkonzentration ist möglicherweise der Befund von Iida ${ }^{30)}$ zurückzuführen, daB das Halbstufenpotential von Nitrobenzol bei starker Laugekonzentration positiver als bei $\mathrm{pH} 12$ ist.

Die Grenzschicht-Struktur hat zwei wesentliche Einflüsse auf die sekundäre Durchtrittsgeschwindigkeit ${ }^{11}$. Ihr erster Einfluss besteht in einer Änderung der vorgela- 
Tabelle 5. Werte fer formalen Geschwindigkeitskonstanten $k_{0}$ und des Durchtrittsfaktors $\boldsymbol{\alpha}$ für den Sekundärprozess (II) sowie Halbstufenpotentiale, ohne und mit Inhibitor, für $m$-Nitrochlorbenzol in wässriger Lösung (10\% Methanol, 0,02n $\mathrm{NaOH})$ bei verschiedenen Ionenstärken $I$ (eingestellt mit $\mathrm{KCl}$ ).

\begin{tabular}{c|c|c|c|c|c|c}
\hline \multirow{2}{*}{$I$} & \multirow{2}{*}{$N_{\varepsilon}$} & \multicolumn{2}{|c|}{ ohne Inhibitor } & \multicolumn{3}{|c}{ mit $0,05 \%$ Campher } \\
\cline { 3 - 7 }$(\mathrm{mol} / 1)$ & $(\mathrm{V}, \mathrm{GKE})$ & $\log k_{0}$ & $\varepsilon_{1 / 2}$ & $\log k_{0}$ & $\begin{array}{c}\varepsilon_{1 / 2} \\
(2 . \mathrm{Stufe})\end{array}$ & $\alpha$ \\
& & $\left(k_{0}\right.$ in cm/sec $)$ & $(\mathrm{V}, \mathrm{GKE})$ & $\left(k_{0}\right.$ in $\left.\mathrm{cm} / \mathrm{sec}\right)$ & $(\mathrm{V}, \mathrm{GKE})$ & \\
\hline 0,1 & $-0,635$ & $-2,10$ & $-0,605$ & $-5,87$ & $-0,975$ & 0,51 \\
0,3 & $-0,633$ & $-1,71$ & $-0,59$ & $-5,59$ & $-0,92$ & 0,55 \\
1,0 & $-0,630$ & $-1,305$ & $-0,575$ & $-5,24$ & $-0,87$ & 0,58 \\
\hline
\end{tabular}

gerten Gleichgewichte : während sie das Gleichgewicht des Primärvorgangs insgesamt zwar unbeeinflusst lässt, verschiebt sie jedoch die drei Einzelgleichgewichte, die dieses zusammensetzen, gegeneinander, und da in die Geschwindigkeitskonstante neben dem Gesamtgleichgewicht noch getrennt das Adsorptionsgleichgewicht des Primärprodukts $(\gamma)$ eingeht, liegt auf diese Weise auch ein beträchtlicher Einfluss auf die Geschwindigkeit vor. Zum zweiten regelt die Grenzschichtstruktur die Bedingungen, unter denen der eigentliche Elektronendurchtritt im adsorbierten Zustand vor sich geht, sodass von ihr auch die Konstante $k$ abhängig ist; dies ist etwa durch das sogenannte " $\phi_{1}$-Potential" zu interpretieren, das vom Aufbau der elektrochemischen Doppelschicht abhängt und seinerseits in die Geschwindigkeitskonstante $k$ eingeht. Die auf Änderungen der Grenzschichtstruktur beruhenden Wirkungen von Lösungsmittel und Leitsalz wurden bereits erwähnt. Der beträchtliche Einfluss der Leitsalzkonzentration sowohl in Abwesenheit wie bei Gegenwart grenzflächenaktiver Stoffe, der im einzelnen quantitativ noch nicht gedeutet ist, geht aus Tab. 5 hervor. Besonders kräftig ist naturgemäss die Wirkung grenzflächenaktiver Stoffe (Inhibitoren), durch welche die Konstante $k_{0}$ um vier bis sechs (und mehr) Grössenordnungen verringert werden kann (vergl. Tab. 3). Diese Wirkung wurde, besonders im Hinblick auf Art und Konzentration des Inhibitors experimentell und theoretisch eingehend untersucht ${ }^{11)}$. Sie beruht sehr wesentlich auf der Verdrängung des Primärprodukts aus der Adsorptionsschicht, also der Verringerung des Adsorptionskoeffizienten $\gamma$. Neuere Untersuchungen über Mechanismus und Kinetik des Primärvorgangs durch Messungen der Faraday-Impedanz ${ }^{16)}$ zeigten, dass z.B. Triphenylphosphinoxid, das ebenso kräftig wirkt wie etwa Campher, den Adsorptionskoeffizienten der Nitroverbindung selbst um etwa zwei Grössenordnungen verringern kann. Besondere Verhältnisse ergeben sich bei Gegenwart grenzflächenaktiver Ionen ${ }^{31}$, da dann der Potentialverlauf in der Grenzschicht anders als bei Adsorption von Neutralmolekülen ist. Zu den Einflüssen der Grenzschichtstruktur gehören auch diejenigen Effekte, die man beim Übergang von der Quecksilber-Tropfelektrode zu Festelektoden beobachtet. Die bei Untersuchungen mit rotierenden Scheibenelektroden ${ }^{14)}$ beobachtete Verringerung von $k_{0}$ an Edelmetallelektroden gegenüber der Quecksilberelektrode beruht sicherlich auf den in diesem Falle (in alkalischen Lösungen) inhibierend wirkenden Deckschichten, zumal der Zusatz von Inhibitoren unter diesen Umständen ohne merklichen Einfluss bleibt.

Bei hinreichend geringer Geschwindigkeitskonstante $k_{0}$ erscheint, wie bereits erwähnt, im Potentialbereich um ${ }^{N_{\varepsilon}}$ nur die einelektronige Primärstufe; die Reduktion bleibt demnach unter polarographischen Bedingungen beim Primärprodukt stehen. Dieses 
besitzt jedoch wegen seines Radikalcharakters nur eine begrenzte Lebensdauer. Während es in nichtwässrigen Lösungsmitteln recht stabil ist ${ }^{23)}$, zerfällt es in wässrig-alkalischen Lösungen, wenn auch mit sehr mässiger Geschwindigkeit ${ }^{32,33}$. Potentiostatische Untersuchungen ${ }^{32}$ zeigten, dass es unter diesen Umständen eine Halbwertszeit von einigen Minuten besitzt und dass der Zerfall in einer Dismutation unter teilweiser Rückbildung der Nitroverbindung und Bildung des Hydroxylamin-Derivats besteht, wie schon früher von uns formuliert ${ }^{8}$, wobei sehr wahrscheinlich auch noch die Nitrosoverbindung zwischenzeitlich auftritt. Die Untersuchungen hierüber sind noch nicht abgeschlossen. Die Stabilität des Radikalanions kann natürlich wesentlich verringert werden, wenn die Lösung andere reduzierbare Substanzen enthält, die-etwa bei Gegenwart von Inhibitoren-an der Elektrode nicht reduziert werden können. In einer Lösungsreaktion werden diese Substanzen dann durch das Radikalanion reduziert, welches seinerseits zur Nitroverbindung zurückoxydiert wird. Bei hinreichend grosser Geschwindigkeit dieser Lösungsreaktion kann es dabei zu einer starken Erhöhung der Primärstufe kommen (katalytische Reduktion der Fremdsubstanzen), wie dies für Perjodat und einige KupferKomplex-Ionen festgestellt wurde ${ }^{10)}$.

\section{Wässrig-neutrale Lösungen bei Inhibitor-Gegenwart}

In wässrigen Lösungen wächst die Geschwindigkeit der eben besprochenen Dismutation des Radikalanions von etwa pH 10 abwärts stark $a^{2,7,8)}$. Die hierdurch bewirkte Rückbildung der Nitroverbindung-die dem eben besprochenen Einfluss reduzierbarer Fremdstoffe ganz analog ist und die man daher als autokatalytischen Effekt bezeichnen kann-erfolgt dann bereits innerhalb der Lebensdauer des polarographischen Tropfens zu einem merklichen Ausmass. Die Stromstärke der Primärstufe steigt daher über diejenige der 1-Elektronen-Reduktion an, bis bei etwa pH 6 die volle 4- (oder 6-) elektronige Stufe erreicht wird. In diesem $\mathrm{pH}$-Bereich wird demnach die langsame Sekundärreduktion teilweise umgangen, und nur der im Laufe des Tropfenlebens auf diese Weise nicht reagierende Anteil erzeugt bei negativeren Potentialen die der Sekundärreduktion zukommende Reststufe. Neuere Untersuchungen ${ }^{\text {;2) }}$ ergaben, dass die Dismutation nach zwei verschiedenen Mechanismen abläuft, wobei entweder eine Reaktion erster oder zweiter Ordnung geschwindigkeitsbestimmend ist. Es hängt daher von pH-Wert und Lösungskonzentration der Nitroverbindung ab, welcher der beiden Mechanismen tragend ist.

Diese Erscheinungen sind nur in Gegenwart von Inhibitoren beobachtbar. In deren Abwesenheit würden-wenn keine weiteren Reaktionsweisen im Spiele wären (vergl. den nächsten Abschnitt)—diese Lösungsreaktionen lediglich eine mässige Vorverlagerung der Gesamtstufe bewirken, da diese ja bereits ihre volle Höhe besitzt.

\section{Saure bis schwach alkalische Lösungen (ohne Inhibitor)}

Tatsächlich rückt die Gesamtstufe in solchen Lösungen jedoch bald unterhalb $\mathrm{pH} 10$ mit sinkendem pH-Wert recht kräftig zu positiveren Potentialen vor, sodass hier ein anderer Mechanismus angenommen werden muss. Wegen des glatten Verlaufs der $\varepsilon_{1 / 2} /$ $\mathrm{pH}^{-G_{e} a d e n^{3}}{ }^{\text {ist }} \mathrm{zu}$ vermuten, dass dieser Mechanismus bis in stark saure Lösungen hinein unverändert bleibt. Zur näheren Untersuchung dieses Mechanismus wurde die Potential- und $\mathrm{pH}$-Abhängigkeit der Stromstärke (zur Vermeidung verschiedenartiger Wirkungen von Puffersubstanzen) in $\mathrm{HCl} / \mathrm{KGl}$-Lösungen ermittelt, und zwar nur bis zu solchen $\mathrm{pH}-$ Werten hinauf, daB noch keine merkliche Verarmung an $\mathrm{H}^{+}$-Ionen eintreten 
konnte. Die Ergebnisse lassen sich formal durch die Beziehung

$$
\frac{i}{i^{*}}=z \cdot \Psi(\chi) \quad \text { mit } \quad \chi=k^{1} \cdot \sqrt{\frac{12 t}{7 D}}
$$

beschreiben. (Zur Bezeichungsweise vergl. Abschnitt II, 1. $z=$ Elektronenzahl des Gesamtvorgangs, also 4 bzw. 6.) Die formale Geschwindigkeitskonstante $k^{1}$ hängt in folgender Weise von der Wasserstoffionen-Konzentration und dem Potential ab:

$$
k^{1}=A \cdot\left[\mathrm{H}^{+}\right]^{\rho_{H}} \cdot 10^{-\rho_{\varepsilon} \frac{\varepsilon}{0,059}}
$$

Für das näher untersuchte p-Nitrochlorbenzol hat der Faktor A den Wert $10^{-4}$, wenn $\varepsilon$ auf die GKE bezogen wird. Die Exponenten $\rho_{H}$ und $\rho_{\varepsilon}$ haben beide einen Wert von ungefähr 1,5. Da unter normalen Bedingungen (Tropfzeit ca. 3 Sekunden) $k^{1}\left(\varepsilon_{1 / 2}\right)=2 \cdot 10^{-3}$ $\mathrm{cm} / \mathrm{sec}$ ist, kann man Gleichung (3) umformen zu

$$
\varepsilon_{1 / 2}=\frac{(\log A+2,7) \cdot 0,059}{\rho_{\varepsilon}}-\frac{\rho_{H} \cdot 0,059}{\rho_{\varepsilon}} \cdot \mathrm{pH}
$$

In der früher ${ }^{2,3)}$ experimentell gefundenen Beziehung

$$
\varepsilon_{1 / 2}=a-b \cdot \mathrm{pH}
$$

ist demnach der Neigungsfaktor $b$ von den beiden Exponenten $\rho_{H}$ und $\rho_{\varepsilon}$ abhängig, wobei Unterschiede zwischen beiden eine mehr oder minder große Abweichung des Faktors $b$ von $0,059 \mathrm{~V} / \mathrm{pH}$ bewirken, während das auf $\mathrm{pH} 0$ extrapolierte Halbstufenpotential $a^{3,4)}$ durch $\rho_{\varepsilon}$ und $A$ festgelegt wird. Legt man die Beziehung (4) zugrunde, so ergeben sichmit der Annahme $\rho_{H} \approx 1,5$-für $A$ Werte zwischen $10^{-2}$ und $10^{-7}$ und für $\rho_{H} / \rho_{\varepsilon}$ solche zwischen 0,8 und 1,3. Der Mechanismus dieser Vorgänge ist im einzelnen noch nicht geklärt. Verschiedene Umstände machen es jedoch wahrscheinlich, dass hierbei neben Durchtrittsreaktionen Protonenübergänge in der Grenzschicht selbst, d.h. im adsorbierten Zustand, verlaufen, wie wir dies bereits in einem anderen Falle vermutet haben ${ }^{34}$ und wie es z.T. auch zur Erklärung widersprüchlicher Ergebnisse bei den bekannten SäureRekombinations-Reaktionen herangezogen wird ${ }^{35}$. Im vorliegenden Falle sprechen hierfür der andere Charakter der funktionellen Zusammenhänge in Gegenwart grenzflächenaktiver Stoffe, die eine solche Grenzflächenreaktion inhibieren würden, ferner die komplizierte Abhängigkeit der Geschwindigkeit von Wasserstoffionen-Konzentration und Potential gemäss Gleichung (3), die sich durch geschwindigkeitsbestimmende Durchtrittsreaktionen und Lösungsreaktionen allein nicht verstehen lässt, sowie auch eine gewisse Abhängigkeit der Parameter in Gleichung (3) von der Depolarisator-Konzentration. Auch die nach Gleichung (4) das Halbstufenpotential bestimmenden thermodynamischen und kinetischen Grössen unterliegen-ebenso wie die Konstante $k_{0}$ in protonenarmen Medien-naturgemäß außer dem $\mathrm{pH}$-Wert Einflüssen von seiten des Lösungsmittels, der Lösungszusammensetzung und der Depolarisator-Konstitution. Letzterer, nämlich die Wirkung weiterer Substituenten am Benzolkern, wurde eingehend untersucht ${ }^{3,4,36}$ und durch die Hammett-Gleichung beschrieben ${ }^{37}$; zweifellos sind diese Zusammenhänge zu einem wesentlichen Teil auf die energetischen Verhältnisse des $\pi$-Elektronensystems zurückzuführen ${ }^{38}$, jedoch scheint uns die Annahme eines Übergangszustandes, in dem bereits alle vier aufzunhemenden Elektronen lokalisiert sind, wie dies von Basu u. Cha uhuri $\mathrm{i}^{39)}$ angesetzt wird, fraglich zu sein; vermutlich liegt der Übergangszustand zwischen der Nitroverbindung und dem ein- (allenfalls zwei-) elektronigen Zwischenprodukt.

In Lösungen mit einem Unterschuss an Wasserstoffionen (vergl. hierzu auch R ü et s c hi 
u. Trümpler ${ }^{40)}$ ) beobachtet man zwei Stufen : die erste ist in ihrer Höhe durch die Diffusion der Wasserstoffionen begrenzt, während die zweite Stufe der Reduktion des restlichen Anteils der Nitroverbindung zukommt, die wie in Abwesenheit von Wasserstoffionen (in alkalischer' Lösung) stattfindet. Die Zugrundelegung der formalen Geschwindigkeitskonstanten nach Gleichung (3) führt wegen des Exponenten $\rho_{H}=3 / 2$ für die $\mathrm{H}^{+}$-Diffusionsstufe auf die gleichen kinetischen Ansätze, wie sie von Koutecký u. $\mathrm{Hanu} \mathrm{s}^{\mathbf{4} 1}$ für bimolekulare Reaktionen abgeleitet wurden. Hieraus lassen sich die Halbstufenpotentiale dieser Stufe berechnen, die mit den experimentell ermittelten übereinstimmen. Liegt anstelle eines Wasserstoffionen-Unterschusses unzureichende Pufferung bei höheren $\mathrm{pH}-$ Werten vor, so erscheint anstelle einer $\mathrm{H}^{+}$-Diffusionsstufe eine kinetische Stufe, deren Höhe durch die Kinetik der Nachlieferung aus dem Puffer-Gleichgewicht bestimmt wird. Aus solchen Untersuchungen konnten Nürnberg, van Riesenberg u. von Stackelberg ${ }^{42}$ die Dissoziationsgeschwindigkeiten von Protonendonatoren ermitteln.

\section{Saure Lösungen bei Gegenwart von Inhibitoren}

Gegenüber dem im vorangehenden Abschnitt besprochenen Reaktionsverlauf ist derjenige in sauren Lösungen $(\mathrm{pH}<5)$ bei Gegenwart von Inhibitoren durchsichtiger. Die Stromspannungskurven lassen sich auch dier durch die Gleichung (2) beschreiben und die $\mathrm{pH}$ - und Potentialabhängigkeit der formalen Konstanten $k$ durch Gleichung (3). In diesem Falle ist jedoch $\rho_{H}=1$ und $\rho_{\varepsilon}=0,7$ (für p-Nitrochlorbenzol bei Gegenwart von $0,1 \%$ Campher; mit $\left.A=5 \cdot 10^{-6}\right)$. Ein solcher Zusammenhang ergibt sich, wenn der primären Elektronenaufnahme ein Dissoziations-Gleichgewicht in der Lösung vorgelagert ist $^{8}$, wobei der Elektronendurchtritt wegen der geringen Gleichgewichtsmenge des Kations als langsamer, geschwindigkeitsbestimmender Schritt auftritt. Dabei muß vorerst offen bleiben, ob das Kation erst eines oder gleich zwei Elektronen aufnimmt;

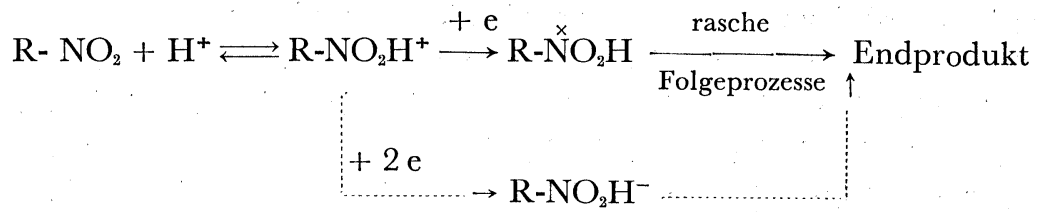

Der Exponent $\rho_{\varepsilon}$ hat dann einfach die Bedeutung des Durchtrittsfaktors $\alpha$. Die Konstante $A$ enthält nun :neben der Geschwindigkeitskonstanten des Elektronendurchtritts (bezogen auf $\varepsilon=0$ ) die Säurekonstante für die Dissoziation des Nitro-Kations. Da letztere nicht bekannt ist, kann die Konstante $A$ nicht aufgetrennt werden. Die $\varepsilon_{1 / 2} / \mathrm{pH}-$ Abhängigkeit ergibt sich analog zu Gleichung (5) mit den obigen Daten und experimentell zu

$$
\varepsilon_{1 / 2}=-0,21-0,085 \cdot \mathrm{pH}
$$

(gegenüber $\varepsilon_{1 / 2}=-0,05-0,06 \cdot \mathrm{pH}$ in inhibitorfreien Lösungen).

Obgleich es-angesichts des sehr unterschiedlichen elektrochemischen Verhaltens organischer Verbindungen - müssig ist, nach einem Prototyp dieser Vorgänge zu suchen, so bietet doch die Reduktion aromatischer Nitroverbindungen eine solche Fülle verschiedenartiger Erscheinungen, dass an ihr die meisten bei organisch-elektrochemischen Prozessen auftretenden Probleme unter einem allgemeineren Gesichtspunkt untersucht werden können. 


\section{Literatur}

1) M. Shikata, J. Agric. Chem. Soc. Japan 1, 81, 533 (1925) ; Trans. Farad. Soc. 21, 42, 53 (1925) ; M. Shikata u. M. Watanabe, J. Agr. Chem. Soc. Japan 4, 924 (1928) ; M. Shikata u. N. Hozaki, Mem. Coll. Agr., Kyoto Imp. Univ. 17, 1, 21, 45 (1931) ; Bull. Agric. Chem. Japan 8, 75 (1932) ; M. Shikata u. I. Tachi, J. Agr. Chem. Soc. Japan 8, 1121 (1932) ; M. Shikata u. E. Taguchi, J. Agr. Chem. Soc. Japan 8, 77, 1225 (1932) ; I. Tachi, Mem. Coll. Agr., Kyoto Imp. Univ. 29, 1 (1934) ; J. Agr. Chem. Soc.Japan 11, 734 (1935) ; Mem. Coll. Agr., Kyoto Imp. Univ. 42, 1 (1938) ; J. Agr. Chem. Soc. Japan 13, 692 (1937).

2) L. Holleck u. H.J. Exner, Proceed. I. Internat. Polarogr. Congr. Prag 1951, Bd. I, S. 97 ; Z. Elektrochem. 56, 46, 677 (1952) ; Z. Naturforsch. 7a, 763 (1951).

3) H.J. Exner, Dissertation, Universität Freiburg (Br.), 1952.

4) L. Holleck, H. Marsen u. H.J. Exner, Z. Naturforsch. 9b, 90 (1954) ; (In dieser Arbeit sind die Unterschriften zu Abb. 5 und 6 vertauscht!).

5) L. Holleck u. H. Schmidt, Z. Elektrochem. 59, 56, 531 (1955).

6) L. Holleck u. G. Perret, Z. Elektrochem. 59, 114 (1955).

7) L. Holleck u. B. Kastening, Naturwissenschaften 43, 298 (1956).

8) B. Kastening u. L. Holleck, Z. Elektrochem. 63, 166 (1959).

9) L. Holleck u. B. Kastening, Z. Elektrochem. 63, 177 (1959) ; Vortragsammlung III. Internat. Kongr. f. Grenzflächenaktive Stoffe, Köln 1960, Bd. B, S. 288.

10) B. Kastening, Naturwissenschaften 47, 443 (1960).

11) B. Kastening u. L. Holleck, Z. Elektrochem. 64, 823 (1960) ; L. Holleck, B. Kastening u. R.D. Williams, Z. Elektrochem. 66, 396 (1962) ; L. Holleck u. B. Kastening, Schriftenreihe "Korrosion", Sonderband, i. Druck.

12) B. Kastening, Naturwissenschaften 49, 130 (1962).

13) L.Holleck u. D. Becher, J. Electroanal. Chem. 4, 321 (1962).

14) L. Holleck, B. Kastening u. H. Vogt, Electrochim. Acta, im Druck.

15) L. Holleck u. D. Jannakoudakis, Z. Naturforsch. 16b, 396 (1961).

16) B. Kastening, H. Gartmann u. L. Holleck, Vortrag 13. GITCE-Tagung, Rom Sept. 1962., Electrochim. Acta,in Vorbereitung.

17) L. Holleck u. D. Jannakoudakis, unveröffentlicht.

18) D. Jannkoudakis, Naturwissenschaften 49, 57 (1962).

19) Ya. I. Turyan, Yu. M. Tyurin u. O.M. Zaytsev, Dokl. Akad. Nauk SSSR 134, 850 (1960).

20) H. Suzuki u. P. Elving, Coll. Czechoslov. Chem. Commun. 25, 3202 (1960).

21) J. Holubek u. J. Volke, Coll. Czechoslov. Chem. Commun. 25, 3286 (1960).

22) W. Kemula u. Z. Kublik, Roczn. Chem. 32, 941 (1958); Anal. Chim. Acta 18, 104 (1958).

23) D.H. Geske u. A.H. Maki, J. Chem. Phys. 33, 825 (1960) ; J. Amer. Chem. Soc. 82, 2671 (1960) ; 83, 1852 (1961).

24) W. Kemula u. R. Sioda, Bull. acad. polon. sci., sér. sci. chim. 10, 107 (1962).

25) J. Heyrovský, F. Sorm u. J. Forejt, Coll. Czechoslov. Chem. Commun. 12, 11 (1947).

26) J.E. Strassner u. P. Delahay, J. Amer. Chem. Soc. 74, 6232 (1952).

27) G. Olson, H.Y. Lee u. R.N. Adams, J. Electroanal. Chem. 2, 396 (1961).

28) A.M. Mirri u. P. Favero, Ricerca sci. 29, 106 (1959).

29) J. Koutecký, Coll. Czechoslov. Chem. Commun. 18, 597 (1953).

30) H. Iida, Chem. Ind. Research Inst., Tokyo 51, 313 (1956) ; H. Iida u. K. Jayahara, Kogyo Kagaku Zasshi 60, 289 (1957).

31) D.J. Pietryzyk u. L.B. Rogers, Anal. Chem. 34, 936 (1962).

32) B. Kastening, unveröffentlicht.

33) P. Ludwig, Z. Galus, H.Y. Lee u. R.N. Adams, Vortrag 13. GITCE-Tagung, Rom. Sept. 1962.

34) B. Kastening, Z. Elektrochem. 64, 82 (1960). 
35) H. Strehlow, Z. Elektrochem. 64, 45 (1960) ; S.G. Mairanovskii, J. Electroanal. Chem. 4, 166 (1962).

36) R. Glicksman u. G.K. Morehouse, J. Electrochem. Soc. 105, 299 (1958) ; V.M. Gorokhovskii u. N.M. Ponomareva, Zhur. Obschei Khim. 29, 3535 (1959).

37) P. Zuman, Coll. Czechosloo. Chem. Commun. 19, 599 (1954) ; J. Tirouflet, Bull. Soc. Chim. France (5), 274 (1956).

38) S. Koide u. I. Tachi, J. Electrochem. Soc. Japan, 23,522 (1955).

39) S. Basu u. J.N. Chaudhuri Nature (London) 180, 1473 (1957)

40) P. Rüetschi u. G. Trümpler, Helv. Chim. Acta 35, 1021, 1486, 1957 (1952).

41) J. Koutecký u. V. Hanuš, Coll. Gzechoslov. Chem. Commun. 20, 124 (1955).

42) H.W. Nürnberg, G. van Riesenbeck u. M.v. Stackelberg, Z. Elektrochem. 64, 130 (1960). 